\title{
Introducción. El género de los sistemas penales juveniles: Debates necesarios
}

\author{
(The Gender of Juvenile Criminal Systems: Necessary Debates)
}

\author{
OÑATI SOCIO-LEGAL SERIES, VOLUME 10, ISSUE 2 (2020), 246-256: EL GÉNERO DE LOS SISTEMAS \\ PENALES JUVENILES: DEBATES NECESARIOS \\ DOI LINK: HTTPS://DOI.ORG/10.35295/OSLS.IISL/0000-0000-0000-1116 \\ RECEIVED 17 MARCH 2020, ACCEPTED 19 MARCH 2020
}

\author{
LAURA LÓPEZ-GALLEGO* (D) \\ CECILIA MONTES-MALDONADO* (iD \\ ENCARNA BODELÓN-GONZÁLEZ*
}

\section{Resumen}

Este número de Oñati Socio-Legal Series, titulado El género de los sistemas penales juveniles: Debates necesarios, es el resultado de las ponencias e intercambios mantenidos en un taller interdisciplinario que tuvo lugar en 2018. Se encuentra integrado por once artículos que discurren sobre las experiencias en justicia penal juvenil dirigidas hacia adolescentes mujeres y otras temáticas vinculadas, a partir de la exposición de resultados de investigaciones e intervenciones realizadas en América Latina (Argentina, Brasil, Chile, México y Uruguay) y el Estado Español (Cataluña, Madrid, País Vasco, Zaragoza). Las discusiones mantenidas en este espacio y los artículos publicados son una muestra más del auge punitivo global que viven las sociedades capitalistas contemporáneas y que podemos observar en los diferentes contextos a través de la extensión de las formas

\footnotetext{
* Profesora Adjunta Dedicación Total del Instituto de Psicología Social de la Facultad de Psicología de la Universidad de la República, Uruguay. Doctora en Psicología por la Universidad Autónoma de Barcelona (2016). Integra el Sistema Nacional de Investigadores de la Agencia Nacional de Investigación e Innovación del Uruguay (ANNI). Datos de contacto: Tristán Narvaja 1674, C.P. 11100, Montevideo, Uruguay. Dirección de email: $\underline{\text { llopez@psico.edu.uy }}$

* Candidata a doctora en Estudios de género: culturas, sociedades y políticas por la Universidad Autónoma de Barcelona. Es licenciada en Psicología (2010) y magíster en Psicología Social (2015) por la Facultad de Psicología (UdelaR). Docente e investigadora del Instituto de Psicología Social de la Facultad de Psicología (UdelaR). Investigadora asociada del Sistema Nacional de Investigadores de la Agencia Nacional de Investigación e Innovación (ANII). Datos de contacto: Universidad Autónoma de Barcelona. Grupo de Investigación Antígona, Edificio B, Facultad de Derecho, Campus de la UAB, 08193 Bellaterra, Barcelona. Teléfono: +34 9358122 74. Dirección de email: cmontes@psico.edu.uy

* Profesora Titular de Filosofía del Derecho, de la Facultad de Derecho de la Universidad Autónoma de Barcelona. Doctora en Derecho, especializada en temas de género y derecho. Directora del grupo de investigación Antígona. Codirectora del posgrado Género e Igualdad de la UAB. Miembro del Institut Interuniversitari d'Estudis de Dones i Génere (iiEDG). Miembro de la Asociación Dones Juristas. Datos de contacto: Universidad Autónoma de Barcelona. Grupo de Investigación Antígona, Edificio B, Facultad de Derecho, Campus de la UAB, 08193 Bellaterra, Barcelona. Dirección de email: encarna.bodelon@uab.cat
} 
de control y alcance de los sistemas penales que se produce sobre la población tanto adulta como joven y adolescente. En este marco, es interesante contextualizar cómo son los tránsitos de las mujeres más jóvenes por los sistemas penales, haciendo énfasis en la necesaria inclusión de una perspectiva de género que pueda comprender las singularidades que forman parte del tratamiento de las mismas en estos sistemas.

\section{Palabras clave}

Adolescentes mujeres; sistemas penales; momento punitivo; criminologías feministas

\section{Abstract}

This issue of Oñati Socio-Legal Series, entitled The Gender of Juvenile Criminal Systems. Necessary Debates, is the result of the communications and exchanges held at a interdisciplinary workshop that took place in 2018. It is composed of eleven articles that discuss experiences in juvenile criminal justice aimed at adolescent women and other related issues, based on the presentation of the results of research and interventions carried out in Latin America (Argentina, Brazil, Chile, Mexico and Uruguay) and the Spanish State (Catalonia, Madrid, Basque Country, Zaragoza). The discussions held in this space and the articles published are one more example of the global punitive expansion that contemporary capitalist societies are experiencing and that we can observe in the different contexts through the extension of the forms of control and scope of the penal systems that are produced on the adult, young and adolescent population. In this framework, it is interesting to contextualize how the transits of younger women through the penal systems are, emphasizing the necessary inclusion of a gender perspective that can understand the singularities that are part of the treatment of women in these systems.

\section{Key words}

Adolescent woman; penal system; punitive moment; feminist criminology 


\section{Índice/Table of contents}

Introducción.

Referencias 


\section{Introducción}

Este número de Oñati Socio-Legal Series, titulado El género de los sistemas penales juveniles: Debates necesarios, es el resultado de las ponencias, intercambios y discusiones que mantuvimos en un taller interdisciplinario que tuvo lugar en 2018. En el mismo nos propusimos reflexionar sobre las experiencias en justicia penal juvenil dirigidas hacia adolescentes mujeres y otras temáticas vinculadas, a partir de la exposición de resultados de investigaciones e intervenciones realizadas en América Latina (Argentina, Brasil, Chile, México y Uruguay) y el Estado Español (Cataluña, Madrid, País Vasco, Zaragoza).

Las discusiones mantenidas durante el taller y los artículos aquí publicados son una expresión más del auge punitivo global que viven las sociedades capitalistas contemporáneas y que podemos observar en los diferentes contextos a través de la extensión de las formas de control y alcance de los sistemas penales sobre la población tanto adulta como joven y adolescente. Extensión que se traduce en aumento y endurecimiento de las penas, creación de nuevos delitos, intenciones de bajar la edad de imputabilidad penal, y más (Wacquant 2012, Fassin 2018, Garland 2018). Así atravesamos lo que Didier Fassin (2018) nombra como momento punitivo, el cual tiene su máxima expresión en el aumento de la población carcelaria a nivel mundial. El castigo inicialmente avizorado como un remedio al problema de la criminalidad, actualmente emerge como el gran desafío a analizar.

En este marco societal, es interesante contextualizar cómo son los tránsitos de las mujeres más jóvenes por los sistemas penales (Almeda 2002, 2017, Carlen y Worral 2004, Antony 2007, Centro de Estudios Legales y Sociales (CELS) 2011, Almeda Samaranch y Di Nella 2017), haciendo énfasis en la necesaria inclusión de una perspectiva de género que pueda comprender las singularidades que forman parte del tratamiento de las mismas en estos sistemas (Galeotti 2013, Bodelón y Aedo 2015, López-Gallego 2016, Montes-Maldonado et al. 2018). La apuesta que realizamos en este número monográfico es poder pensar en términos de interseccionalidad (Crenshaw 2004), los sistemas sexo-género (Rubin 1975) se traman con otras dimensiones de la desigualdad como pueden ser la edad, etnia, clase social, configurando una compleja matriz de dominación.

Como enfatiza April Bernard (2013) el enfoque de la interseccionalidad se presenta como una alternativa que cuestiona la dimensión patológica y de responsabilidad individual históricamente adjudicada a las mujeres que cometen un crimen para resaltar la necesaria comprensión de las estructuras de poder y los sistemas de opresión que circunscriben las experiencias vitales de mujeres localizadas en una intersección de múltiples vulnerabilidades. Así tenemos como en muchas ocasiones determinados procesos de abuso y violencias que se suceden en la vida de estas jóvenes, condicionan la comisión de delitos (Bodelón y Aedo 2015). Investigaciones que aquí se presentan nos muestran la vinculación entre estas dimensiones en lo que hace a la selección y posterior abordaje en los sistemas penales.

Las llamadas Criminologías Feministas (Smart 2000, Bodelón 2003, Pitch 2003, Carlen y Worral 2004) integran las relaciones de género como una forma de dominación presente en los sistemas penales; en tanto éstos se constituyen en tecnologías de género (de Lauretis 1989) que construyen sentidos acerca de la diferenciación de género, sustentados en modelos de convivencia patriarcal. El cómo lo hacen será una de las 
apuestas de las discusiones que componen este número, ¿cómo los sistemas penales funcionan para construir los sistemas de sexo-género?

Como nos señalan algunas investigadoras (Bodelón 2003, Carlen y Worral 2004, Pasko y Chesney-Lind 2013), los sistemas penales construyen relaciones de subordinación por medio de la discriminación y de la igualación. En muchos casos, las reivindicaciones feministas de una justicia igual en el plano formal no se acompañan de tal igualdad, dado que las normas son aplicadas entre personas con fuertes desigualdades sociales. La apuesta por la equidad es otro camino a transitar. Por su parte, las mujeres que son seleccionadas por los sistemas penales obedecen a una doble desviación de la normatividad penal y de género, lo que determina un abordaje que vincula la tríada feminización, domesticación y medicalización (Carlen y Worral 2004).

Asimismo, en la esfera jurídica, las mujeres han aparecido equiparadas a los niños/as, en función de requerimientos de normalización, tutela y protección. Los delitos destacados son aquellos que atentan contra el orden de las familias y la moralidad pública, destacando sus roles de cónyuge, madre y/o hija, fundamentalmente como cuidadoras. Las construcciones asociadas a la criminalidad de las mujeres surgen de forma temprana vinculadas a lo sexual, a través de la figura de la prostituta se encarna la desviación de lo femenino por excelencia, lo que anteriormente fueron las brujas, condenadas por copular con el diablo (Larrandart 2000, Fries y Matus 2000).

En el caso de las adolescentes se conjugan dos condiciones, ser mujeres y menores de edad, lo que comporta procesos de control y vulneración mayores, asociados a la tutela infantilizada. El cuerpo, en tanto que espacio privilegiado de castigo se hará presente, haciendo foco en el terreno del disciplinamiento de las sexualidades (Pasko 2010, LópezGallego et al. 2018). Maternidades en prisión, relaciones homoafectivas, visitas íntimas, derechos sexuales y reproductivos, procesos de sexualización y denegación de la violencia sexual son algunos de los temas que abordan los diversos artículos que componen este número.

En este sentido, este número monográfico cuenta con once producciones. A través de los primeros siete artículos se profundiza en las singularidades del funcionamiento de los sistemas penales juveniles, seguido por un artículo que analiza cómo la categoría de género influye en las decisiones que se disponen en la Justicia Penal Juvenil. Luego se encuentra un artículo que se focaliza en las experiencias de la detención policial que viven las mujeres. $\mathrm{Y}$ por último, contamos con dos aportaciones que buscan explicaciones en torno a los elementos que influyen en la comisión de infracciones y/o delitos.

Detallamos así, de forma sucinta cada artículo e invitamos a detenerse en la lectura detenida de cada uno de ellos:

El texto de Jalusa Silva De Arruda y Natasha María Wangen Krahn nos brinda una profusa revisión bibliográfica que sistematiza investigaciones llevadas adelante en el marco de programas de posgrado (Maestría y Doctorado) de Brasil en relación al sistema de justicia juvenil. Especialmente aquellas dedicadas al estudio de la ejecución de medidas socioeducativas de mujeres adolescentes y que siguen perspectivas de género y/o enfoques feministas. A partir de esta revisión realizan una sistematización, mapeo y discusión académica desde diversas áreas del conocimiento de la totalidad de 51 
artículos de tesis defendidas entre 2012 y 2017. Todos los estudios versan sobre la experiencia de las niñas en la ejecución de la medida socioeducativa y la intersección entre las categorías sociales de género, raza, clase y generación. Entre las principales temáticas abordadas se encuentran: efectos de la institucionalización y del encarcelamiento; procesos de toma de decisiones en ámbito judicial; diferencias entre las prácticas de castigo destinadas a varones y mujeres; en varios trabajos provenientes de psicología se estudian las motivaciones y justificaciones para la comisión de infracciones; e, investigaciones relacionadas con la identidad de género y el ejercicio de la sexualidad en los centros de implementación de las medidas socioeducativas.

En el artículo en portugués escrito por Andrea Coelho Scisleski y Giovana Barbieri Galeano, se discute el proceso de invisibilización de las adolescentes mujeres en el contexto de la ejecución de medidas judiciales en Brasil. Utilizando la caja de herramientas propuesta por Michel Foucault para analizar las relaciones de saber-poder, reflexionan acerca del modelo hegemónico masculino que atraviesa la concepción de las medidas socioeducativas propuestas en el Estatuto da Criança e do Adolescente (Lei no 8.069). A través de una metodología cualitativa de investigación, que combina el estudio de documentos elaborados para la formación de operadores técnicos y de visitas realizadas en un establecimientos de ejecución de medidas socioeducativas en medio abierto en la ciudad de Campo Grande, Brasil, las autoras analizan cómo las adolescentes mujeres son entendidas en función de mandatos hegemónicos acerca del "ser mujer", priorizando su condición de madres y prohibiendo la visita íntima y las relaciones homoafectivas. Como conclusión, las autoras hacen énfasis en la necesidad de problematizar las prácticas y los saberes que sustentan las prácticas de los profesionales que trabajan en la ejecución de las políticas públicas para jóvenes en conflicto con la ley, con el objetivo prioritario de luchar por las garantías de derechos que existen actualmente, y que no siempre se cumplen.

Laura López-Gallego presenta una discusión acerca de las prácticas de castigo generizadas en el marco del Sistema Penal Juvenil Uruguayo (SPJU). La autora argumenta acerca del género de los procesos de criminalización y las particularidades presentes en los tránsitos de las mujeres por los sistemas penales juveniles. Tomando los aportes de las criminologías feministas, analiza los sistemas penales juveniles en clave mujer, planteando que las mujeres seleccionadas han cometido una doble desviación: penal y de género. El material empírico está compuesto por los resultados de dos investigaciones cualitativas y etnográficas realizadas en Uruguay, en el marco de la ejecución de medidas judiciales privativas y no privativas de libertad respectivamente. En relación al corpus producido la autora discute dos líneas analíticas que traman las singularidades del tránsito de las adolescentes mujeres por el SPJU: la (re)construcción de la domesticidad perdida y la gestión de los cuerpos y las sexualidades. En estos marcos, se vuelve prioritario situar las relaciones de género como un principio organizador de las prácticas y discursos de los sistemas penales juveniles, de modo de poder analizar las tecnologías que operan fijando sentidos unívocos de feminidad.

Por su parte, Nathali Estevez Grillo y Carla Cristina García realizan un aporte sustantivo para la comprensión de las vivencias de las niñas acerca de la privación de libertad en San Pablo, Brasil. El énfasis de estas investigadoras está en las tácticas de resistencia que ponen en juego estas niñas en la cotidianidad de su vida presas. Como 
estrategia metodológica recurren a la investigación-acción participante y a las metodologías feministas, a través de observaciones y entrevistas analizan en forma detallada la vida cotidiana en el Centro de Atención Socioeducativo al Adolescente CASA Chiquinha Gonzaga. La criminología crítica y la interseccionalidad del feminismo negro están presentes en las discusiones que presentan estas autoras, las categorías de raza, género y clase constituyen los pilares de vulnerabilidad que estructuran la condición de las mujeres en las cárceles. A través de tres ejes; musicalidades, materialidades y afecto describen las tácticas de resistencia a las opresiones, considerando a estas adolescentes como sujetas epistemológicas productoras de cultura. En este sentido, las resistencias son pensadas como un conjunto de tácticas de producción de vida que las niñas operan con el fin de facilitar y hacer menos penoso el tiempo de internamiento.

Cecilia Montes-Maldonado propone en su artículo un análisis sobre las medidas socioeducativas privativas de libertad destinadas a adolescentes en el Sistema Penal Juvenil Uruguayo. El acento radica en la importancia de analizar los componentes de la ética del cuidado en agentes a cargo de la custodia de adolescentes mujeres y varones en los centros destinados a la privación de libertad. La investigación es cualitativa y muestra un estudio de caso único, en profundidad y con enfoque etnográfico en los centros donde se implementan medidas judiciales de protección a niños, niñas y adolescentes y medidas privativas de libertad a adolescentes. La autora destaca tres aspectos que dan cuenta de las interacciones cotidianas entre agentes a cargo de la custodia y adolescentes como son: las trayectorias y vivencias de los/as agentes institucionales en la institución; las formas en que estos agentes interpretan y definen los significados de las medidas socioeducativas y los modos de significar la población que atienden. A partir de este análisis destaca la relevancia de reorientar la ejecución de las medidas socioeducativas donde el enfoque de los cuidados se encuentre en el centro y colabore para mejorar las condiciones en que la población adolescente reside en el sistema.

El trabajo presentado por María Cristina Gonçalves Vicentin y Daniel Adolpho Dantin Assis muestra el debate brasileño sobre los derechos sexuales y reproductivos de niños, niñas y adolescentes, haciendo foco en las niñas que transitan por el sistema de justicia. A través de tres apartados analíticos analizan los derechos sexuales y reproductivos en su condición de derechos políticos, relacionados al concepto de autonomía. En el primer apartado, describen algunos elementos del debate brasileño y los marcos legales sobre los derechos sexuales y reproductivos; en una segunda parte presentan las tensiones relativas al ejercicio de esos derechos en el campo de las prácticas institucionales con foco en las unidades de internación de niñas. El desafío de ampliar la noción de autonomía, conjugada con la posibilidad de pensar la protección y la responsabilidad en clave de interdependencia, constituye un desafío para los derechos humanos de niños, niñas y adolescentes. Finalmente proponen articular los derechos sexuales y reproductivos y la salud sexual y reproductiva como un campo estratégico para pensar derechos políticos de niños, niñas y adolescentes en la medida en que puede conducir a avances significativos en la concepción y ampliación del contenido de esos derechos, especialmente para los adolescentes que encuentran mayores obstáculos para su ejercicio. 
Natasha María Wangen Krahn, Jalusa Silva De Arruda y Jussara Carneiro Costa nos aportan un análisis muy valioso respecto al acceso al derecho de mantener visitas conyugales durante la privación de libertad de mujeres y adolescentes en el estado de Bahía en Brasil. Desde una investigación exploratoria que utiliza diversas fuentes de información muestran en los resultados cómo las mujeres se enfrentan a diversas restricciones, obstáculos y procesos burocráticos para acceder al derecho de las visitas conyugales. Del análisis de estas restricciones que imponen las condiciones institucionales se observa la fuerte preocupación institucional por el control de las visitas conyugales en todo sentido, la falta de espacio físico adecuado, diversos procedimientos burocráticos que enlentecen los permisos y otras acciones que dan cuenta del atravesamiento de cuestiones morales y de control que pone en funcionamiento la institución. Destacan a su vez que estas restricciones no se producen de igual manera en los establecimientos destinados a varones.

El texto que presentan Ricardo Gallego Córcoles y Raquel Bartolomé Gutiérrez nos ilustra acerca de cómo pensar las cuestiones de género y su incidencia en la toma de decisiones de la Justicia Juvenil. A través de un diseño de investigación descriptivo y de naturaleza retrospectiva, trabajan con una muestra de 166 jóvenes expedientados (127 chicos y 39 chicas) en el marco institucional del Juzgado de Menores de Guadalajara. Como dato interesante, todas las profesionales que han tenido incidencia en las decisiones son mujeres, circunstancia ésta en la que vale la pena detenerse para analizar la discusión de los resultados. En éstos, los/as autores/as muestran que no existen diferencias significativas entre chicos y chicas, en todas las decisiones tomadas en las fases del procedimiento judicial. La hipótesis planteada en relación al trato benévolo que reciben las chicas no pudo ser comprobada, lo que abre la discusión acerca del trato equitativo o igual en el marco de las profundas diferencias sociales de las sociedades patriarcales en las que vivimos.

El artículo de Estíbaliz de Miguel Calvo y Lohitzune Zuloaga Lojo es un aporte relevante de una temática muy poco abordada en el Estado Español y también a nivel internacional, como es la experiencia de la detención policial a mujeres. Aquí profundizan en el estudio de la Comunidad Autónoma de Euskadi donde desde la perspectiva de género analizan los perfiles sociodemográficos de las de mujeres imputadas o detenidas y los niveles de cumplimiento de los derechos que corresponden durante la detención.

Gracias a un original uso de la perspectiva interseccional, situacional e interactiva analizan cómo interactúan de forma compleja los ejes de clase, género, etnia y cultura en las experiencias de detención policial. Así, en los resultados destacan que entre las imputadas y detenidas existe una sobrerrepresentación de extranjeras y de mujeres pertenecientes a grupos atravesados por varios ejes de desigualdad. Además, concluyen que las mujeres detenidas expresan que sus derechos han sido respetados y que no han recibido malos tratos. Sin embargo, las investigadoras afirman la importancia de profundizar en este aspecto dadas las dificultades para expresar aspectos relacionados al maltrato psicológico.

María José Bernuz Beneitez y María González Álvarez analizan en su artículo la relación entre el desarrollo moral de la población adolescente y sus comportamientos delictivos (autorreportados), clasificando las conductas en función de si son contra la 
propiedad, las personas, el orden o la salud pública. Realizaron una encuesta (2012) en la Comunidad Autónoma de Aragón a una muestra de 1.762 adolescentes de entre 14 y 18 años, de los cuales 895 son chicas (50,8\%), y 867, chicos (49,2\%). Como aspectos a resaltar de este estudio, las autoras argumentan que se confirman algunas de la hipótesis de factores de riesgo, en ese sentido los chicos reportan más comportamientos delictivos que las chicas, situación que podría explicarse por una socialización más centrada en el cuidado y la empatía. Otros aspectos como la mayor edad, la legitimación de la violencia y la sumisión incrementan significativamente el riesgo de delinquir.

Por último, el artículo que nos aporta Ricardo Rodríguez Luna se dedica a estudiar las relaciones entre género, edad y violencia en los homicidios que se suceden en México. Para problematizar estas relaciones muestra primero una serie de datos estadísticos que dan cuenta del grado de victimización y de responsabilidad penal del delito de homicidio de los jóvenes mexicanos. Luego expone tres enfoques criminológicos que han abordado el problema de la sobrerrepresentatividad masculina en el delito, los enfoques positivista, sociológico y de la diferencia sexual. Y en último término, analiza las acciones emprendidas por el gobierno mexicano con el fin de prevenir el homicidio de varones jóvenes. Concluye afirmando la urgencia de la implementación de estrategias públicas que incidan en el ordenamiento de género que asocia masculinidad y diversas formas de expresión de la violencia.

\section{Referencias}

Almeda, E., 2002. Corregir y castigar: El ayer y hoy de las cárceles de mujeres. Serie General universitaria 19. Barcelona: Bellaterra.

Almeda Samaranch, E., 2017. Criminologías feministas, investigación y cárceles de mujeres en España. Papers. Revista de Sociologia [en línea], 102(2), 151-81.

Disponible en: https://doi.org/10.5565/rev/papers.2334 [Con acceso el 23 de marzo de 2020].

Almeda Samaranch, E., y Di Nella, D., 2017. Mujeres y cárceles en América Latina. Perspectivas críticas y feministas. Papers. Revista de Sociologia [en línea], 102(2), 183. Disponible en: https://doi.org/10.5565/rev/papers.2335 [Con acceso el 23 de marzo de 2020].

Antony, C., 2007. Mujeres invisibles: Las cárceles femeninas en América Latina. Nueva Sociedad, n. ${ }^{\circ} 208,73-85$.

Bernard, A., 2013. The Intersectional Alternative: Explaining Female Criminality. Feminist Criminology [en línea], 8(1), 3-19. Disponible en: https://doi.org/10.1177/1557085112445304 [Con acceso el 23 de marzo de 2020].

Bodelón, E., 2003. Género y sistema penal: los derechos de las mujeres en el sistema penal. En: R. Bergalli, ed., Sistemas penales y problemas sociales. Valencia: Tirant lo Blanch, 451-86.

Bodelón, E., y Aedo, M., 2015. Las niñas en el Sistema de Justicia Penal. Anales de la Cátedra Francisco Suárez [en línea], 49, 219-236. Disponible en: http://revistaseug.ugr.es/index.php/acfs/article/view/3283 [Con acceso el 23 de marzo de 2020]. 
Carlen, P., y Worral, A., 2004. Analysing Women's Imprisonment. Londres: Willan.

Centro de Estudios Legales y Sociales, 2011. Mujeres en prisión: Los alcances del castigo. Buenos Aires: Siglo XXI.

Crenshaw, K., 2004. Interseccionalidad: una herramienta para la justicia de género y la justicia económica. Derechos de las mujeres y cambio económico, n. ${ }^{\circ}$ 9, 1-10.

De Lauretis, T., 1989. Technologies of Gender: Essays on Theory, Film and Fiction. Londres: Macmillan Press.

Fassin, D., 2018. Castigar: Una pasión contemporánea. Buenos Aires: Adriana Hidalgo.

Fries, L., y Matus, V., 2000. La ley hace al delito. Santiago de Chile: LOM / La Morada.

Galeotti, R., 2013. Adolescentes infractoras: Discursos y prácticas del Sistema Penal Juvenil Uruguayo. Montevideo: Psicolibros-Waslala.

Garland, D., 2018. Theoretical Advances and Problems in the Sociology of Punishment. Punishment \& Society [en línea], 20(1), 8-33. Disponible en: https://doi.org/10.1177/1462474517737274 [Con acceso el 23 de marzo de 2020].

Larrandart, L., 2000. Control social, derecho penal y género. En: H. Birgin, ed., Las trampas del poder punitivo: El género del derecho penal. Buenos Aires: Biblos, 85-109.

López-Gallego, L., 2016. Prácticas de control socio-penal. Dispositivo psi pericial y adolescentes mujeres en el Sistema Penal Juvenil Uruguayo. Tesis Doctoral. Universitat Autònoma de Barcelona.

López-Gallego, L., Galeotti-Galmés, R., y Montes-Maldonado, C., 2018. Gestión de las sexualidades en los sistemas penales: las adolescentes mujeres. Revista Latinoamericana de Ciencias Sociales, Niñez y Juventud [en línea], 16(1), 413-26.

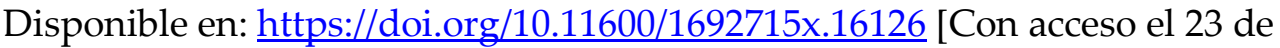
marzo de 2020].

Montes-Maldonado, C., López-Gallego, L., y Galeotti-Galmes, R., 2018. Adolescentes mujeres y medidas no privativas de libertad: narrativas de una experiencia etnográfica. Psicoperspectivas. Individuo y Sociedad [en línea], 17(2). Disponible en: https://doi.org/10.5027/psicoperspectivas-Vol17-Issue2-fulltext-1212

Pasko, L., 2010. Damaged Daughters: The History of Girls' Sexuality and the Juvenile System. The Journal of Criminal Law and Criminology, 1099-1130.

Pasko, L., y Chesney-Lind, M., eds., 2013. The Female Offender: Girls, Women, and Crime. $3^{\underline{a}}$ ed. Thousand Oaks: Sage.

Pitch, T., 2003. Responsabilidades limitadas: Actores, conflictos y justicia penal. Buenos Aires: Ad-Hoc.

Rubin, G., 1975. The Traffic in Women: Notes toward a Political Economy of Sex. En: R. Reiter, ed., Toward an Anthropology of Women. Nueva York: Monthly Review Press. pp. 157-210.

Smart, C., 2000. La teoría feminista y el discurso jurídico. En: H. Birgin, ed., El Derecho en el Género y el Género en el Derecho. Buenos Aires: Biblos, 31-71. 
Wacquant, L., 2012. Castigar a los pobres: El gobierno neoliberal de la inseguridad social. Barcelona: Gedisa. 